\title{
Surgical monotherapy may be a suitable therapeutic strategy for advanced collecting (Bellini) duct carcinoma: A case report and literature review
}

\author{
LIANG ZHU ${ }^{1}$, ZIJIAN WANG ${ }^{1}$, CIZHONG PAN $^{1}$, RAN PENG $^{2}$, XIONG WEI $^{1}$ and ZHAOHUI ZHONG ${ }^{1}$ \\ Departments of ${ }^{1}$ Urology and ${ }^{2}$ Cardiology, The Second Xiangya Hospital, \\ Central South University, Changsha, Hunan 410011, P.R. China
}

Received March 15, 2015; Accepted April 19, 2016

DOI: $10.3892 /$ etm.2016.3402

\begin{abstract}
Collecting duct carcinoma (CDC), which is also known as Bellini duct carcinoma, accounts for less than $1 \%$ of all renal cell carcinoma, which has a poor prognosis. Predominantly, clinicians recognize this disease due to past experience and case reports, and a standard treatment is yet to be established. The present case report describes a 57-year-old male patient with CDC who was successfully treated with a left laparoscopic radical nephrectomy without any adjuvant therapy and compares the present patient's clinical presentation with previously reported cases. By analyzing the differences between these cases, the findings of the present case report and literature review suggested that surgery alone remains the only suitable choice for patients with advanced CDC.
\end{abstract}

\section{Introduction}

Collecting duct carcinoma (CDC), which is also known as Bellini duct carcinoma, is a type of kidney cancer that originates in the papillary duct of the kidney. Accounting for $<1 \%$ of all renal cell carcinoma (RCC) cases, patients with CDC have a poor prognosis (1). Predominantly, clinicians recognize this disease due to past experience and case reports, and a standard treatment is yet to be established. However, since CDC is diagnosed based on pathological examination, surgery is thus inevitable in the majority of such cases. To the best of our knowledge, the majority of patients with CDC that present at an advanced stage will succumb to the disease within one

Correspondence to: Dr Xiong Wei, Department of Urology, The Second Xiangya Hospital, Central South University, Ward 1, 139 Renming Road, Changsha, Hunan 410011, P.R. China E-mail: xiongwei1609@163.com

Abbreviations: CDC, collecting duct carcinoma; RCC, renal cell carcinoma; RN, radical nephrectomy; CT, computerized tomography

Key words: carcinoma, renal cell, Bellini, therapeutics year post-surgery, regardless of whether they receive or deny adjuvant therapies (1-7).

According to the literature, the majority of CDC cases have been high grade, advanced stage and unresponsive to conventional therapies (8). As shown in studied cases in the present study (Table I), the majority of CDC cases survived between 4 and 9 months post-operatively, and the majority of such patients present at an advanced stage, regardless of whether they receive or deny adjuvant therapies. The current study reports a CDC case treated with surgical monotherapy, and the post-surgery survival conditions are compared with other reported cases. The aim of the study is to determine whether surgical monotherapy is a suitable therapeutic strategy for patients with advanced CDC.

\section{Case report}

A 57-year-old male presented at the Department of Urology at The Second Xiangya Hospital, Central South University (Changsha, China) in July 2013 with intermittent painless gross hematuria that lasted for two weeks. The results of laboratory tests demonstrated that blood electrolytes were within the normal ranges, and renal and liver function were normal. An X-ray was performed, which showed the patient had a clear chest. A subsequent computerized tomography (CT) scan demonstrated a rough bordered tumor measuring $7.1 \times 6.0 \mathrm{~cm}$ on the left kidney, which was heterogeneously enhanced, and multiple enlarged lymph nodes were detected in the retroperitoneal space, particularly in the area surrounding the left renal artery (Fig. 1). No other abnormalities or metastasis were detected. According to 2009 TNM classification system (8), the patient was diagnosed as clinical stage T2bN2M0. Written informed consent was obtained from the patient prior to publication.

Following diagnosis, a left laparoscopic radical nephrectomy (RN) was performed. During surgery, a group of enlarged lymph nodes were found fused with one another near the left renal hilum, and were firmly fixed to the psoas major and the aorta descendens. Notably, the left renal artery was engulfed by this nodular mass and it was impossible to remove all of these lymph nodes due to their infiltration; therefore, only partial resection of these lymph nodes was 
Table I. Summary of patient tumor characteristics and survival duration.

\begin{tabular}{|c|c|c|c|c|c|}
\hline Author, year & $\begin{array}{c}\text { TNM } \\
\text { classification }^{\mathrm{a}}\end{array}$ & Stage $^{a}$ & Treatment & $\begin{array}{l}\text { Survival duration } \\
\quad(\text { months })^{\mathrm{b}}\end{array}$ & Refs. \\
\hline \multicolumn{6}{|l|}{ Surgery alone } \\
\hline The present case & $\mathrm{T} 2 \mathrm{bN} 2 \mathrm{M} 0$ & IV & RN & 9 & - \\
\hline \multirow[t]{2}{*}{ Bansal et al, 2012} & T4N0M0 & IV & $\mathrm{RN}$ & 9 & (2) \\
\hline & T1bN0M0 & I & $\mathrm{RN}$ & 9 & \\
\hline \multirow[t]{5}{*}{ Méjean et al, 2003} & T3N2M1 & IV & $\mathrm{RN}$ & Very short & (3) \\
\hline & T3N2M1 & IV & $\mathrm{RN}$ & Very short & \\
\hline & T3N2M1 & IV & $\mathrm{RN}$ & Very short & \\
\hline & T3N2M1 & IV & $\mathrm{RN}$ & 6 & \\
\hline & T3N2M1 & IV & $\mathrm{RN}$ & 9 & \\
\hline \multirow[t]{2}{*}{ Chao et al, 2002} & T3aN1M1 & IV & $\mathrm{RN}$ & 7 & $(10)$ \\
\hline & T4N2M1 & IV & $\mathrm{RN}$ & 13 & \\
\hline \multicolumn{6}{|c|}{ Surgery plus chemotherapy } \\
\hline Milowsky et al, 2002 & T4N2M0 & IV & $\mathrm{RN}+\mathrm{Dox}+\mathrm{Gem}$ & 10 & (4) \\
\hline \multirow[t]{2}{*}{ Orsola et al, 2005} & T4N1MO & IV & $\mathrm{RN}+\mathrm{Dox}+\mathrm{Gem}$ & Mean 5.6 & $(5)$ \\
\hline & T3aN1M0 & III & $\mathrm{RN}+\mathrm{Dox}+\mathrm{Gem}$ & & \\
\hline \multicolumn{6}{|c|}{ Surgery plus combined therapy } \\
\hline \multirow[t]{2}{*}{ Husillos et al, 2011} & T1N0M1 & IV & $\mathrm{RN}+\mathrm{Tem}$ & 4 & (6) \\
\hline & $\mathrm{T} 3 \mathrm{bN} 2 \mathrm{M} 1$ & IV & $\mathrm{RN}+\mathrm{Sun}$ & 7 & \\
\hline \multirow[t]{5}{*}{ Procopio et al, 2012} & TxN1-2Mx & III-IV & $\mathrm{RN}+$ Sor & Mean 4 & (7) \\
\hline & TxN1-2Mx & III-IV & $\mathrm{RN}+$ Sor & & \\
\hline & TxN1-2Mx & III-IV & $\mathrm{RN}+$ Sor & & \\
\hline & TxN1-2Mx & III-IV & $\mathrm{RN}+$ Sun & & \\
\hline & TxN1-2Mx & III-IV & $\mathrm{RN}+\mathrm{Tem}$ & & \\
\hline \multirow[t]{2}{*}{ Chao et al, 2002} & T3aN0M1 & IV & $\mathrm{RN}+\mathrm{Pac}+\mathrm{Car}$ & 9 & $(10)$ \\
\hline & T3aN0M1 & IV & $\mathrm{RN}+\mathrm{Pac}+\mathrm{Car}$ & 17 & \\
\hline
\end{tabular}

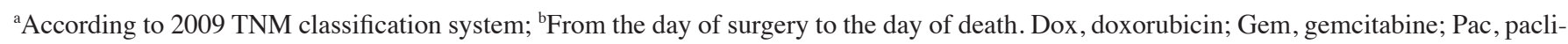
taxel; Car, carboplatin; Tem, temsirolimus; Sun, sunitinib; Sor, sorafenib.
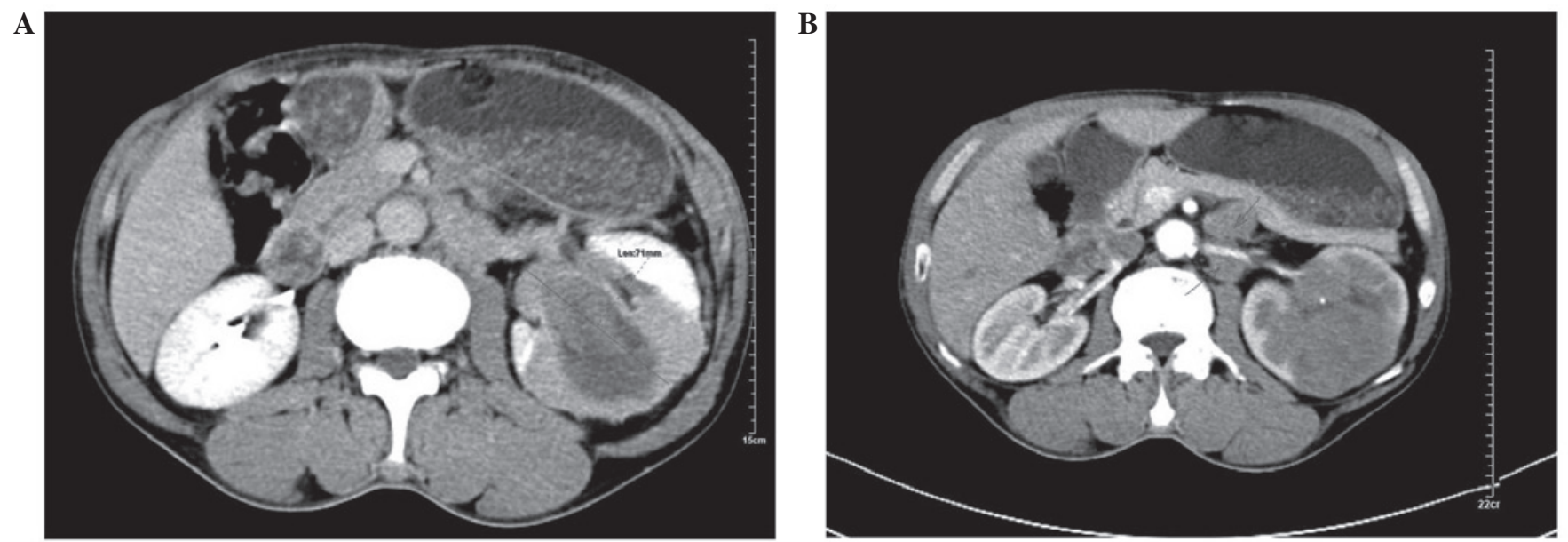

Figure 1. Computed tomography scanning demonstrated (A) a heterogeneously enhanced tumor with a maximum diameter of $71 \mathrm{~mm}$ and (B) enlarged retroperitoneal lymph nodes that engulfed the left renal artery.

performed for biopsy. The affected left kidney was successfully removed, and on gross examination a reddish-brown tumor measuring $6.8 \times 6.5 \times 2.5 \mathrm{~cm}^{3}$ was found in the upper pole of the kidney. A postoperative pathological diagnosis of renal collecting (Bellini) duct carcinoma was made as the lymph nodes were positive for cancer, according to the following criteria: i) Pre-operative CT scan shown multiple enlarged lymph nodes; ii) perinephric lymph nodes enlarged 
and hardened during surgery; iii) post-operative pathologic examination confirmed that the resected lymph nodes were cancerous. Pathological diagnosis was performed at the Department of Pathology of The Second Xiangya Hospital, Central South University. Tissue was formalin-fixed and the core lesion was embedded in paraffin, cut into $\sim 5 \mu \mathrm{m}$ sections, dried at room temperature overnight, then de-paraffinized and rehydrated for staining. Immunohistochemical analysis was performed using the following antibodies purchased from Abcam (Cambridge, UK): Ki-67 (rabbit polyclonal antibody; cat. no. ab15580,), epithelial membrane antigen (rabbit polyclonal; cat. no. ab139390) and vimentin (mouse polyclonal; cat. no. ab7752); and cytokeratin 7 (CK7; mouse polyclonal; cat. no. ab82253), smooth muscle actin, renal cell carcinoma (RCC; rabbit polyclonal; cat. no. ab152111), cluster of differentiation 10 (mouse monoclonal; cat. no. ab951), prostate specific antigen (mouse monoclonal; cat. no. ab49395), human melanoma black 45 (HMB45; mouse monoclonal; cat. no. ab787), thyroid transcription factor-1 (mouse monoclonal; cat. no. ab72876), S100 (rabbit polyclonal; cat. no. ab15520), hematopoietic cell kinase (HCK; rabbit polyclonal; cat. no. ab32860) and lymphocyte-specific protein tyrosine kinase-negative (rabbit monoclonal; cat. no. ab32149). SignalStain Boost ICH Detection Reagent (Cell Signaling Technology, Inc., Danvers, MA, USA) was used.

The patient discharged from hospital 5 days post-surgery. When the final diagnosis was made, the patient was offered adjuvant chemotherapy but he refused due to the fear of the adverse effects of chemotherapy and, more importantly, the uncertainty of the current regimens. The patient was followed-up three months post-surgery and he reported no discomfort. Since the gross hematuria had been alleviated, the patient was quite relieved and satisfied with his condition. Six months after surgery, on his second follow-up, the patient did not complain of any discomfort. Abdominal CT scanning demonstrated enlarged residual lymph nodes in the retroperitoneal space that were in the same position as the previously CT scan.

Two months later, at eight months post-surgery, the patient presented at The Second Xiangya Hospital again complaining a loss of appetite. Chest X-ray analysis demonstrated a nodular mass located near the right lung hilus. Lung metastasis was considered and a chest CT scan was recommended; however, the patient refused to complete further CT examination for personal reasons, nor was he willing to receive further therapies.

Although the patient did attend our hospital again, consultations were performed over the telephone. One month later, the patient developed dyspnea due to hydrothorax, and thoracentesis was performed several times at The First People Hospital and Yueyang (Yueyang, China) to aid his breathing. Palliative treatment was administered and the patient succumbed to respiratory failure nine months and two days post-surgery.

\section{Discussion}

CDC is a highly malignant tumor which accounts for $<1 \%$ of all RCC cases (1). Due to its rarity, the majority of clinicians only recognize this disease due to past experience and case reports. To the best of our knowledge, the largest CDC case series to date includes 81 patients (9), and the majority of patients with
CDC present with advanced staged disease, predominantly with lymphadenopathy or metastasis. However, no consensus has been reached concerning treatment protocols. The present case report and review aimed to summarize and evaluate patient's experiences with CDC.

Surgery remains the most effective treatment for patients with renal cancer, even in patients with advanced disease. Since $\mathrm{CDC}$ is diagnosed on the basis of pathology, imaging examinations and clinical manifestations prior to surgery are unable to accurately distinguish CDC from other types of kidney tumors; thus surgery is inevitable in the majority of cases. Regrettably, no survival data of patients with advanced kidney cancer without surgery is available in the literature.

In a previous study by Tokuda et al (9), $98.8 \%$ of cases underwent surgery as primary treatment, including radica nephrectomy, partial nephrectomy, total nephroureterectomy and biopsy of the metastatic site. These surgeries were performed in $88.9,2.5,7.4$ and $1.2 \%$ of cases, respectively. Furthermore, Bansal et al (2) reported two cases of CDC (T4N0M0 and T1bN0M0), both of whom received RN alone and survived for 9 months. In another previous report, two patients with CDC (T3aN1M1 and T4N2M1) also received RN and survived for 7 and 13 months post-surgery, respectively (10). In a study performed by Méjean et al (3), one of three patients succumbed to stage T3N2M1 CDC shortly after surgery, whereas the remaining two cases survived for 6 and 9 months following surgery, respectively. The patient patient survived for 9 months and 2 days following RN surgery, which appears to be consistent with the previous cases (Table I).

The majority of CDC cases reported in the literature were unresponsive to conventional therapies. In the report published by Milowsky et al (4), a patient with T4N2M0 CDC was treated with RN plus adjuvant chemotherapy (doxorubicin + gemcitabine) and survived for 10 months post-surgery. Furthermore, Orsola et al (5) reported two cases (T4N1M0 and $\mathrm{T} 3 \mathrm{aN} 1 \mathrm{M} 0$ ) who received the same treatment as the patient described by Milowsky et al (4); however, the mean survival time was only 5.6 months post-surgery (5). Chao et al (10) described two stage T3aN0M1 CDC cases, both of whom were treated with RN plus adjuvant chemotherapy (paclitaxel + carboplatin); the patients succumbed to CDC 9 and 17 months post-surgery, respectively. These previous cases demonstrated that, in addition to the side effects and economic burden of chemotherapy, the improvement in patient survival induced by adjuvant chemotherapy is unsatisfactory.

Targeted therapy is another promising therapeutic option for patients with advanced RCC; however, according to the literature, its curative effect for CDC remains poor. Husillos et al (6) reported two CDC cases at stages T1N0M2 and T3bN2M1, respectively, whom were treated with RN plus targeted therapy (temsirolimus and sunitinib) and survived for 4 and 7 months post-RN, respectively. Furthermore, in another previous report, five CDC patients with nodal involvement were treated with $\mathrm{RN}$ plus targeted therapy [sorafenib $(\mathrm{n}=3)$; sunitinib $(\mathrm{n}=1) ; 1$ temsirolimus $(n=1)]$ as first-line treatment, and, after suffering from early progression, the mean survival time of these patients was 4 months (7).

In previous cases, chemotherapy has been combined with targeted therapy. Staehler et al (11) reported two stage T3aN2M0 CDC cases treated with RN plus combined therapy 
(first line, gemcitabine + cisplatin; second line, sunitinib); both patients succumbed to the disease 8 months after the initial diagnosis. However, Barrascout et al (12) described a patient with T3aN0m0 CDC treated with RN plus combined therapy (gemcitabine, cisplatin and bevacizumab) who survived $>35$ months following the initial diagnosis.

It is cursory to state that all patients with $\mathrm{CDC}$ have a poor prognosis, as there have been reports of patients with CDC who have survived $>5$ years with controlled disease $(4,7,13)$. Notably, none of these patients exhibited lymphadenopathy or metastasis prior to surgery and all received different therapies. Conversely, all advance staged CDCs patients, particularly those with nodal involvements, suffered from a poor prognosis regardless of the therapy they received, which suggests that adjuvant therapy may not be an independent factor.

In conclusion, following case comparison, no superiority in the type of adjuvant therapy was detected in the present case report and literature review. Therefore, the patient's quality of life and economic factors should be taken into consideration until novel adjuvant therapies are proven effective. The authors of the present study hypothesize that surgical monotherapy may be the most suitable treatment for patients with advanced CDC.

\section{References}

1. McDougal WS, Wein AJ, Kavoussi LR, Novick AC, Partin AW, Peters CA, Ramchandani P (eds): Campbell-Walsh Urology. In: Malignant Renal Tumors. Campbell SC and Lane BR (eds). Chapter 49. 10th edition. Elsevier Health Sciences, USA, pp1436, 2011.

2. Bansal P, Kumar S, Mittal N and Kundu AK: Collecting duct carcinoma: A rare renal tumor. Saudi J Kidney Dis Transpl 23: 810-812, 2012.
3. Méjean A, Rouprêt M, Larousserie F, Hopirtean V, Thiounn N and Dufour B: Is there a place for radical nephrectomy in the presence of metastatic collecting duct (Bellini) carcinoma? J Urol 169: 1287-1290, 2003.

4. Milowsky MI, Rosmarin A, Tickoo SK, Papanicolaou N and Nanus D: Active chemotherapy for collecting duct carcinoma of the kidney. Cancer 94: 111-116, 2002.

5. Orsola A, Trias I, Raventós CX, Español I, Cecchini L and Orsola I: Renal collecting (Bellini) duct carcinoma displays similar characteristics to upper tract urothelial cell carcinoma. Urology 65: 49-54, 2005.

6. Husillos A, Herranz-Amo F, Subirá D, Lledó E, Molina-Escudero R and Hernández-Fernández C: Collecting duct renal cell carcinoma. Actas Urol Esp 35: 368--371, 2011 (In Spanish).

7. Procopio G, Verzoni E, Iacovelli R, Colecchia M, Torelli T and Mariani L: Is there a role for targeted therapies in the collecting ducts of Bellini carcinoma? Efficacy data from a retrospective analysis of 7 cases. Clin Exp Nephrol 16: 464-467, 2012.

8. Sobin LH, Gospodarowicz MK and Wittekind C (eds): TNM Classification of Malignant Tumors. 7th edition. Blackwell Publishing, Ltd., Oxford, pp336, 2009.

9. Tokuda N, Naito S, Matsuzaki O, Nagashima Y, Ozono S and Igarashi T; Japanese Society of Renal Cancer: Collecting duct (Bellini duct) renal cell carcinoma: A nationwide survey in Japan. J Urol 176: 40-43; discussion 43, 2006.

10. Chao D, Zisman A, Pantuck AJ, Gitlitz BJ, Freedland SJ, Said JW, Figlin RA and Belldegrun AS: Collecting duct renal cell carcinoma: Clinical study of a rare tumor. J Urol 167: 71-74, 2002.

11. Staehler M, Schöppler G, Haseke N, Stadler T, Karl A, Siebels M, Ihrler S and Stief CG: Carcinoma of the collecting ducts of Bellini of the kidney: Adjuvant chemotherapy followed by multikinase inhibition with sunitinib. Clin Genitourin Cancer 7: 58-61, 2009.

12. Barrascout E, Beuselinck B, Ayllon J, Bättig B, Moch H, Teghom C and Oudard S: Complete remission of pulmonary metastases of Bellini duct carcinoma with cisplatin, gemcitabine and bevacizumab. Am J Case Rep 13: 1-2, 2012.

13. Chua ME, Olondriz K, Maniwa M, Mendoza J and Castillo J: Collecting duct of Bellini renal carcinoma with psoas muscle recurrence: A case report and review of literature. Can Urol Assoc J 8: E167-E171, 2014. 